\title{
Nota Farmacológica: ¿Son seguros los antidepresivos en el embarazo? Haciendo foco sobre los IRSS
}

\author{
Are antidepressants safe in pregnancy? A Focus on SSRIs. Therapeutics Letter, January-February 2010
}

Traducido con autorizacion de "Therapeutics Initiative" (The University of British Columbia) Disponible en URL: http://ti.ubc.ca/PDF/76.pdf

\begin{abstract}
Catherine recibía $20 \mathrm{mg}$ de paroxetina diarios hace tres años atrás por síntomas depresivos que aparecieron tras un divorcio complicado. El tratamiento solo duró dos semanas ya que la medicación la hacía sentirse nerviosa. Su depresión se resolvió a las seis semanas. Catherine se volvió a casar y junto a su marido estaban considerando tener hijos cuando descubrió que estaba embarazada de dos meses. Ahora está sufriendo los mismos síntomas de depresión que experimentó hace tres años. Ante ésta situación, consulta a su médico de familia sobre la posibilidad de tomar un antidepresivo durante el embarazo. ¿Que dice la evidencia al respecto?
\end{abstract}

En la Columbia Británica la toma de inhibidores selectivos de la recaptación de serotonina (IRSS) se duplicó, pasando de un $2 \%$ en 1998 a un $5 \%$ en $2001^{1}$. Su uso también se duplicó en Quebec a lo largo del mismo período de tiempo ${ }^{2}$, y en siete sistemas de medicina prepaga de EE.UU. $(n=118.935)$ su uso aumentó de un $2 \%$ en 1996 a un $8 \%$ en $2004-5^{3}$.

\begin{abstract}
Las mujeres embarazadas ¿tienen mayor riesgo de sufrir depresión?

El embarazo no se asocia con mayor riesgo de sufrir depresión $^{4}$. Una revisión sistemática estimó que la prevalencia de depresión en el embarazo oscila entre un 3,1 a 4,9\% por trimestre. A su vez, un $7,5 \%$ de las mujeres embarazadas sufren episodios nuevos de depresión. Una encuesta representativa de todo EE.UU. encontró valores similares de depresión en mujeres embarazadas y no embarazadas: 5,6 contra $8,1 \%{ }^{5}$.
\end{abstract}

\begin{abstract}
La depresión durante el embarazo ¿conduce a algún tipo de daño?

La depresión durante el embarazo se asocia a aumento de la tasa de prematurez, bajo peso al nacer, bajos puntaje de APGAR, necesidad de cuidados intensivos neonatales, hipertensión gestacional y pre-eclampsia, cesárea, depresión post-parto, desnutrición, tabaquismo y consumo de alcohol y drogas ilegales ${ }^{6}$. Sin embrago, los estudios no logran establecer si la depresión conduce hacia algún tipo de daño, o si estos resultados desfavorables se deben a una asociación entre depresión y factores como la pobreza, condiciones de vida precarias, inadecuada contención social o embarazos previos dificultosos.
\end{abstract}

¿Cuáles son los beneficios que aportan los IRSS para la madre durante el embarazo?

No existe evidencia de beneficio proveniente de ensayos clínicos aleatorizados y controlados (ECA); no se han realizado ECA que comparen la toma de IRSS durante el embarazo contra el tratamiento no farmacológico, tratamiento con otros antidepresivos o directamente sin tratamiento. Un estudio de cohortes frecuentemente citado $(n=201)$, comparó mujeres embarazadas que continuaban la toma de antidepresivos durante el embarazo contra mujeres que discontinuaron la medicación por un tiempo mayor o igual a una semana ${ }^{7}$. El promedio de duración de la depresión de la cohorte era mayor a 15 años. El $43 \%$ de las participantes sufrieron recaídas de su sintomatología, de la cuales el $31 \%$ pertenecía al grupo que continuó tomando los antidepresivos y el $68 \%$ al grupo que cesó. Los autores no reportaron síntomas de abstinencia y no se describe ningún protocolo en relación a una disminución gradual de las dosis. Además, por el momento de aparición de las recaídas, probablemente muchos síntomas interpretados como recaídas eran en realidad síntomas de abstinencia.
Tampoco fueron reportados resultados en relación a la calidad de vida, partos, funcionalidad, efectos adversos serios y totalidad de efectos adversos.

El sesgo de publicación ha exagerado la eficacia de los IRSS aproximadamente en un tercio en la población adulta no embarazada ${ }^{8}$. Los IRSS no superan los resultados del placebo a nivel clínico en la depresión leve y moderada`. Un meta-análisis que comparaba tratamiento farmacológico contra no farmacológico para la depresión, no encontró diferencias en general; los IRSS mostraron ser levemente más efectivos que la psicoterapia, pero sin significancia clínica; la psicoterapia resultó igual de efectiva para la depresión severa y leve ${ }^{10}$.

\section{¿Cuáles son los beneficios de los IRSS para el niño?}

Ocho estudios de cohortes compararon el tratamiento con antidepresivos contra ningún tratamiento en mujeres embarazadas con depresión. Tres de estos estudios consistieron en análisis de bases de datos públicos: dos en la Columbia Británica ${ }^{1},{ }^{11}$ y uno en un sistema de medicina prepaga de EE.UU ${ }^{12}$. Los otros cinco estudios eran de tipo clínico $(n=44 \text { a 107) })^{13,14,15,16,17}$. Estos estudios no proveyeron ningún tipo de evidencia que demostrara que los antidepresivos mejoraran la salud infantil. La mortalidad no fue estudiada. La tasa de internación en cuidados intensivos no difirió significativamente en los distintos grupos ( $n=268$; cuatro estudios): $16,6 \%$ en el grupo tratado con IRSS contra $8,6 \%$ en el grupo sin tratamiento. Tampoco hubo diferencias en el peso al nacer ( $n=268$; seis estudios): los promedios fueron $3,36 \mathrm{~kg}$ en el grupo IRSS contra $3,53 \mathrm{~kg}$ en el grupo sin tratamiento. Hubo más partos pretérmino en el grupo IRSS ( $n=548$; tres estudios): 11,8 contra $4,7 \%$, RR=2,2 (IC95\% 1,2 a 4,1), NND=14; y un mayor número de niños sufrieron dificultad respiratoria al nacer ( $n=1743$; dos estudios): 12,4 contra $8,2 \%, R R=1,6($ IC95\% 1,2 a 2,1), NND=24.

¿Cuáles son los daños que puede sufrir el feto asociado al uso de IRSS durante el embarazo?

- Abortos espontáneos: la exposición a IRSS se asoció a más abortos espontáneos en un análisis de cohortes de mujeres que contactaron un servicio de información sobre teratogenia $(n=1874)^{18}$ y en un meta-análisis de seis estudios de cohortes ${ }^{19}$.

- Malformaciones cardíacas: La paroxetina es el único IRSS sujeto a alertas regulatorios sobre el riesgo de teratogenia. Un meta-análisis del laboratorio GSK, realizado sobre 14 estudios observacionales hasta el 2008 (diez estudios de cohortes y cuatro estudios de casos y controles), encontró un incremento en las malformaciones cardíacas, $R R=1,5$ (IC95\% 1,2 a 1,8), NND aprox. 20, y en las malformaciones totales, $R R=1,2$ $(\text { IC95\% 1,1 a 1,4) })^{20}$

Sin embargo, parece haber probablemente un efecto de clase. Durante un seguimiento de ocho años de todos los nacimientos ocurridos en Dinamarca, un $0,9 \%$ de las mujeres que recibieron dos o más prescripciones para IRSS durante el embarazo $(n=1370)$ tuvieron hijos con malformaciones cardíacas septales, contra un $0,5 \%$ de eventos en el grupo de mujeres que no los recibieron $(n=493.113), O R=2,0 \quad(I C 95 \% 1,1$ a 3,5$)$, $\mathrm{NND}=246^{21}$. Las mujeres expuestas a más de un IRSS tuvieron mayor riesgo que las no expuestas, OR=4,7 (IC95\% 1,7 a $12,7)$, NND=62. Para esto se tuvo en cuenta el uso de citalopram, sertralina y cualquier otro IRSS.

En un centro médico terciario de Israel, se investigaron todos los soplos cardíacos infantiles mediante ecocardiografía. Se 
encontraron malformaciones cardíacas en el 3,4\% de los niños expuestos a IRSS durante el primer trimestre de embarazo, contra un $1,6 \%$ de los no expuestos, RR=2,2 (IC95\% 1,1 a $4,4)^{22}$. Un estudio holandés mostró que los niños con exposición prenatal continua a IRSS $(n=197)$ sufrieron mas cirugías cardíacas que los no expuestos ( $n=36.998)$, OR=5,6 (IC95\% 1,9 a 16,3) a pesar de haberse realizado similar cantidad de estudios complementarios ${ }^{23}$.

Dos estudios compararon la tasa de malformaciones cardíacas infantiles en hijos de mujeres con diagnóstico de depresión que tomaban IRSS, contra mujeres que no los tomaban. No se encontró ninguna diferencia $(n=10.878) R R=1,3 \quad(I C 95 \%$ 0,7$2,1) 11,12$.

- Hipertensión pulmonar persistente del recién nacido (HPPRN): La HPPRN ocurre en alrededor de uno cada 100 nacimientos y es potencialmente fatal ${ }^{24}$. Un estudio de casos y controles encontró una de la tasa de HPPRN cinco veces mayor en infantes expuestos a IRSS después de la semana 20 del embarazo ${ }^{25}$. En un análisis efectuado a partir del registro de nacimientos ocurridos en Suecia, tambiénse encontró un incremento del riesgo de sufrir HPPRN en hijos de mujeres expuestas a IRSS en las últimas etapas del embarazo ( $\mathrm{n}=2.350 / 831.324)$ HPPRN: RR=3,6 $(\text { IC95\% 1,2-8,3) })^{26}$.

Dos estudios estadounidenses no lograron confirmar éstos últimos datos, pero ninguno poseía el poder adecuado para mostrar una diferencia significativa ${ }^{27,28}$.

- Mala adaptación neonatal: síntomas como irritabilidad, llanto persistente, temblor, intranquilidad, dificultades para alimentarse y trastornos del sueño fueron reportados en el 20 a $30 \%$ de los niños expuestos a IRSS en el tercer trimestre ${ }^{29,30}$. Los síntomas suelen ser leves y transitorios, pero también pueden presentarse convulsiones, requiriendo algunos niños de intubación. La concentración sérica de paroxetina resultó más alta en niños con mala adaptación neonatal en comparación con aquellos sin síntomas, a pesar de haber recibido sus madres dosis similares ${ }^{31}$. Todavía no se conoce la tasa de efectos adversos a largo plazo a nivel cognitivo y del desarrollo.

\section{Tratamientos no farmacológicos}

Los tratamientos no farmacológicos no suelen probarse en el embarazo. Un ECA $(n=38)$ evaluó el tratamiento con psicoterapia a lo largo de 16 semanas $^{32}$. Otro $(n=150)$ evaluó el efecto del tratamiento con acupuntura ${ }^{33}$. Ambos demostraron una reducción en los síntomas de depresión.

\section{Conclusiones e implicancias clínicas}

- No existe evidencia que sugiera que los IRSS mejoren la salud materna o del infante. Sin embargo existe evidencia suficiente que los IRSS representan un riesgo para ambos. Por lo tanto los daños exceden los beneficios.

- Los tratamientos no farmacológicos como las terapias conductuales o la psicoterapia no han mostrado evidencia de efectividad, pero tampoco son riesgosas para el feto. Un argumento en contra de su aplicación suele ser la baja disponibilidad de estos recursos, pero en estos casos el mismo es irrelevante ya que se trata de una población pequeña y de alta prioridad.

- Si una mujer quisiera dejar de tomar IRSS durante el embarazo, idealmente se debería disminuir la dosis en forma gradual a lo largo de una semana como mínimo para reducir los síntomas de abstinencia.

- El ejercicio, la contención social, una adecuada higiene del sueño y una buena nutrición son importantes para todas las mujeres embarazadas, incluyendo aquellas con depresión.

$\mathrm{NND=Número} \mathrm{necesario} \mathrm{para} \mathrm{dañar;} R R=$ Riesgo relativo; $I C=$ Intervalo de confianza; OR=Odds ratio

\section{Referencias}

1. Oberlander TF, Warburton W, Misri S et al. Neonatal outcomes after prenatal exposure to selective serotonin reuptake inhibitor antidepressants and maternal depression using population-based linked health data. Arch Gen Psychiatry 2006; 63(8):898-906.

2. Ramos E, Oraichi D, Rey E et al. Prevalence and predictors of antidepressant use in a cohort of pregnant women. BJOG 2007; 114(9):1055-1064

3. Andrade SE, Raebel MA, Brown J, et al. Use of antidepressant medications during pregnancy: a multisite study. Am J Obstet Gynecol 2008; $198(2): 194 . e 1-e 5$.

4. Gaynes BN, Gavin N, Meltzer-Brody S et al. Perinatal depression: prevalence, screening accuracy, and screening outcomes. Evidence Report/Technology Assessment No. 119. AHRQ Publication No. 05- E006-2. Rockville, MD: Agency for Healthcare Research and Quality. February 2005.

5. Vesga-Lopez O, Blanco C, Keyes K et al. Psychiatric disorders in pregnant and postpartum women in the United States. Arch Gen Psych 2008; 65(7):805-815.

6. Bonari L, Pinto N, Ahn E et al. Perinatal risks of untreated depression during pregnancy. Can J Psychiatry 2004; 49(11):726-35.

7. Cohen LS, Altshuler LL, Harlow BL et al. Relapse of major depression during pregnancy in women who maintain or discontinue antidepressant treatment. JAMA 2006; 295(5): 499-507.

8. Turner EH, Mathews AM, Linardatos E et al. Selective publication of antidepressant trials and its influence on apparent efficacy. N Engl J Med 2008; 358(3):252-60.

9. Kirsch I, Deacon BJ, Huedo-Medina TB et al. Initial severity and antidepressant benefits:a meta-analysis of data submitted to the Food and Drug Administration. PLoS Med 2008; 5(2):e45.

Para obtener la lista complete de referencias, incluyendo las citas 10 a 33, dirigirse a: http://ti.ubc.ca/letter76\#1

El borrador de ésta nota farmacológica fue sometido a revisión por 55 expertos y médicos de atención primaria con el objeto de corregir cualquier imprecisión y asegurar que la información fuese concisa y relevante para los lectores

La nota terapéutica representa un resumen de la evidencia obtenida de ensayos clínicos farmacológicos evaluados críticamente, que puede aplicarse a pacientes similares a los incluidos en esos ensayos pero no ser generalizable a cada paciente en particular. Nuestro objetivo es evaluar la efectividad de nuestras actividades educativas utilizando la red PharmaCare/PharmaNet, sin identificar médicos individuales, farmacias o pacientes. Therapeutics Initiative es financiado por el Ministerio de Salud de la Columbia Britanica a través de un subsidio a la Universidad de la Columbia Británica. Therapeutics Initiative provee consejos basados en la evidencia sobre terapéutica farmacológica, y no es responsable de la formulación o adjudicación de las políticas provinciales de medicamentos. 